\title{
Redevenir généraliste après une grave crise existentielle
}

\author{
Auteur: un collègue concerné, co-auteur: Mirjam Tannera
}

a Dr med., spécialiste en psychiatrie et psychothérapie, Comité de direction de ReMed

\begin{abstract}
Un médecin de famille passionné reprend son activité médicale après avoir traversé une grave crise personnelle et professionnelle. Il raconte comment il a retrouvé confiance en lui grâce au soutien du réseau ReMed. Le nouveau projet de soutien intitulé «Stage de réinsertion en cabinet médical» permet aux médecins qui ont vécu une période difficile de reprendre pied dans la profession.
\end{abstract}

«Suite à mon burnout, je n'étais plus en mesure d'exercer en tant que médecin de famille. J'ai non seulement dû renoncer à une activité professionnelle qui me comblait, mais également à ma vie de famille, à mes enfants et à ma maison. Les fondements de ma vie personnelle et professionnelle ont ainsi volé en éclats.

Les entretiens proposés par ReMed offrent non seulement des conseils, mais également un soutien pour élaborer un projet professionnel.

J'ai obtenu de l'aide, notamment au travers du réseau de soutien ReMed avec lequel j'avais des entretiens réguliers par téléphone, qui m'ont offert, dans un premier temps, conseil et soutien et m'ont ensuite permis de planifier ma réinsertion professionnelle et de re-

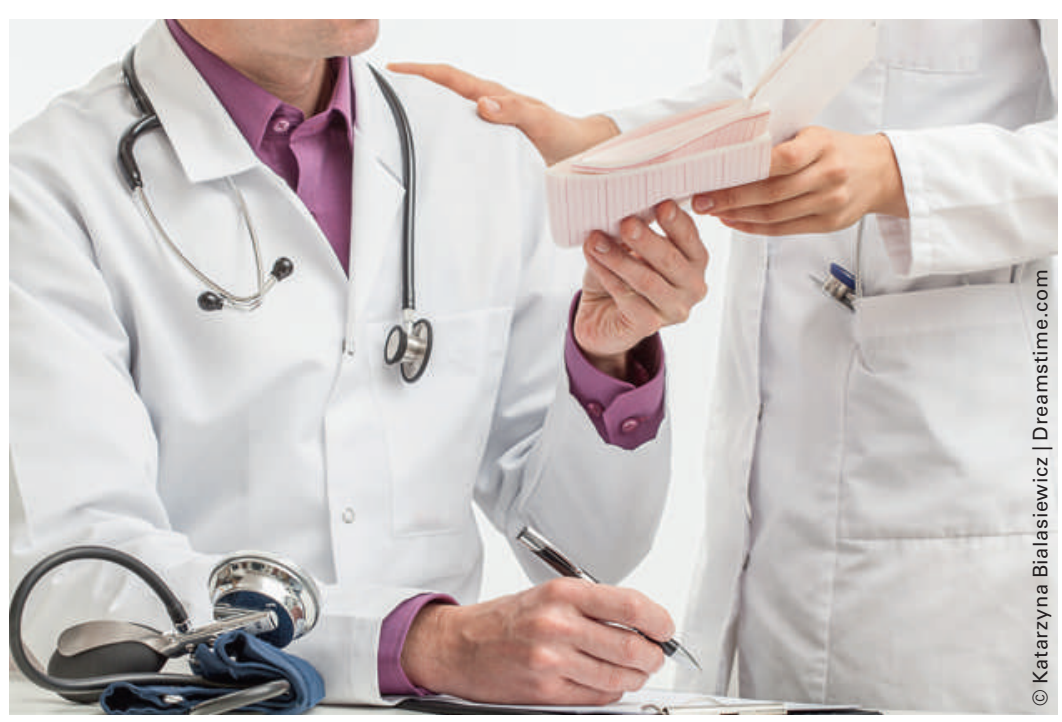

Reprendre son activité en étant bien accompagné grâce à un stage de réinsertion auprès d'un mentor en pratique privée.

\section{ReMed est à vos côtés}

Avez-vous, ou une personne de votre entourage, besoin d'un soutien professionnel? Si tel est le cas, n'hésitez pas à contacter ReMed, le réseau de soutien pour médecins. ReMed respecte le secret médical et vous accompagne de manière compétente en cas de crise personnelle ou professionnelle. Après une première prise de contact, l'équipe de conseillers de ReMed vous répond dans les 72 heures. Pour de plus amples informations: www.swiss-remed.ch, help[at]swiss-remed.ch, tél.: 0800073633.

prendre mon activité. Les indemnités journalières versées par la Caisse maladie des médecins suisses ont été d'un grand secours, mais ce chômage ou plutôt cette incapacité de travail chronique m'a beaucoup pesé et je me sentais seul et déprimé. Le divorce, la séparation d'avec mes enfants, le manque de contacts sociaux à mon nouveau domicile se sont aussi révélés difficiles. Bien qu'à l'époque, je n'étais pas en mesure de travailler comme généraliste, cette activité épanouissante me manquait.

\section{Catalogué et sans perspectives}

Au printemps 2010, je me suis senti mieux et j'ai postulé auprès d'assureurs et d'autres médecins de famille en leur demandant à pouvoir travailler dans leur cabinet dans le but de me réinsérer professionnellement. Les réponses négatives que j'ai reçues de la plupart de mes confrères ont engendré beaucoup de frustration. Ils considéraient vraisemblablement le fait que je n'aie pas travaillé pendant un an pour des raisons de santé comme trop risqué. Ce manque de confiance a eu un impact négatif sur mon estime de moi-même et je me sentais réduit à ma maladie. 
Le fait de ne pas travailler, le manque de structures quotidiennes et l'isolement social et professionnel m'ont fait replonger en dépression six mois plus tard et j'ai dû être hospitalisé. J'ai suivi un traitement médicamenteux et des séances de psychothérapie qui m’ont

\section{Progressivement, j'ai traité mes patients avec de plus en plus d'autonomie et mes craintes, notamment celle de ne pas être à la hauteur, se sont dissipées.}

permis d'aller enfin mieux. J'ai repostulé et après deux ans d'incapacité de travail, la chance m'a souri. Un médecin de ma connaissance m'a proposé de travailler à l'essai à 50\% dans son cabinet avec le soutien financier de la Caisse maladie des médecins.

\section{«Stage de réinsertion en cabinet médical»: recherchons mentors et médecins intéressés}

Suite à une absence pour raison de crise personnelle ou professionnelle, les médecins peuvent se sentir désécurisés et craindre de reprendre leur activité en pratique privée. Grâce à un stage en cabinet médical auprès d'un mentor, ils peuvent reprendre confiance en eux. Durant ce stage de plusieurs semaines, le médecin exerce sous la supervision du mentor, retrouve confiance en ses capacités et peut déterminer ses éventuelles lacunes dans le domaine médical et social et dans la manière de gérer un cabinet.

Une telle coopération présente des avantages pour les mentors en raison de la pénurie croissante de médecins de famille, notamment en termes de suppléance et de reprise ultérieure du cabinet. Cela permet en outre d'assurer la qualité et les qualifications de l'entreprise. Dans l'idéal, les mentors ont de l'expérience en tant que formateurs, sont bien implantés et familiers des offres de ReMed.

Cherchez-vous un médecin en pratique privée pour accomplir un stage de réinsertion? Etes-vous intéressé à accompagner un confrère pour lui permettre de se réinsérer professionnellement? Un projet-pilote est en cours jusqu'à fin 2017. Si vous êtes intéressé ou concerné, n'hésitez pas à nous contacter par courriel à info[at]swiss-remed.ch ou par téléphone au 031 3591200.

\section{Groupes d'intervision: rencontres en 2016}

A la demande des médecins, ReMed a lancé en 2009 des séances d'intervision entre confrères. Depuis, le réseau met régulièrement sur pied des rencontres entre pairs (6-10 participants, 2-3 fois par an). Les participants évoquent ensemble les questions concernant le mentorat, le coaching, les conseils, les traitements et d'autres aspects de l'accompagnement entre médecins (questions juridiques, d'assurance, etc.). Si vous souhaitez participer à une séance, n'hésitez pas à prendre contact avec nous. Personne à contacter: Peter Birchler, par téléphone au 0443420910 ou par courriel à peter.birchler[at] hin.ch.

Prochaines rencontres:

27.10.16 Hôtel Plaza Zurich, 14h-18h

17.11.16 Hôtel Plaza Zurich, 14h-18h

\section{Retour progressif dans le monde du travail}

$\mathrm{Au}$ début, je traitais les patients sous la supervision $\mathrm{du}$ détenteur du cabinet, puis progressivement, de manière autonome. Mes craintes, en particulier celle de ne pas être à la hauteur, se sont dissipées. Après deux mois, je me sentais à nouveau compétent, tant du point de vue humain que professionnel. J'étais donc prêt à assumer un emploi et j'ai trouvé un poste de généraliste à $70 \%$ dans une permanence médicale en ville. Grâce au soutien de mon employeur, j'ai pu reprendre ma profession avec beaucoup de gratitude. Il me tient à cœur que mes collègues médecins qui se trouvent dans une situation difficile puissent rapidement recevoir de l'aide. Il est par ailleurs essentiel qu'on leur permette, s'ils le souhaitent, de reprendre leur activité professionnelle même après une longue absence pour cause de maladie. Il me semble également important que cette réinsertion soit encadrée par d'autres médecins et pas par les procédures difficiles, impersonnelles et coûteuses de l'assurance-invalidité. J'estime que nous ne devons pas seulement nous montrer solidaires envers nos patients, mais également envers nos confrères en situation de vulnérabilité tant personnelle que professionnelle.» 